\title{
Hyaluronidase nutzt Arzt und Patient
}

Die Hyaluronidase spaltet die Hyaluronsäure in der Haut und verbessert so die Wirkung der Lokalanästhesie. Die Injektion dieses Enzyms hat sich vor allem bei Blepharoplastiken, der Liposuktion und bei Narbenkorrekturen bewährt.

„Bei kleinen chirurgischen Eingriffen im Bereich der ästhetischen Dermatologie gilt das Enzym Hyaluronidase heute als fester Standard“, so Prof. Alina Fratila, Bonn. Der Schlüssel für den vielfältigen Einsatz der Hyaluronidase (Hylase ${ }^{\circledast}$,Dessau“) liege in ihren gewebeauflockernden Eigenschaften. Das Enzym katalysiert die Spaltung komplexer Kohlenhydrate in der extrazellulären Matrix, sodass deren Viskosität abnimmt. Daraus resultiert eine erhöhte Gewebeflüssigkeit aktiver Substanzen. „Diesen sogenannten SpreadingEffekt macht man sich in der Lokalanästhesie zu Nutze, um die Diffusion und den Wirkeintritt eines Anästhetikums zu beschleunigen und die analgesierte Fläche zu vergrößern“, so Fratila. Der Einsatz von Hyaluronidase sei vorteilhaft für Arzt und Patient; denn der beschleunigte Wirkeintritt des Anästhetikums bedeute für den Operateur eine Zeitersparnis und auch der Patient sei angesichts der geringeren intra- und postoperativen Schmerzen deutlich zufriedener.

„Sehr bewährt hat sich die Hyaluronsäure in der Ophthalmologie sowohl bei Katarakt- und Strabismusoperationen als auch bei Blepharoplastiken“, so Dr. Andreas Britz, Hamburg. Gerade bei diesen Eingriffen ermögliche Hyaluronidase den Verzicht auf eine Vollnarkose. Wegen des positiven Einflusses auf das Operationsergebnis und das Patientenbefinden sei die Hyaluronidase heute der unverzichtbare Standard bei Blepharoplastiken.

Ein besonders großes Potenzial hat die Hyaluronsäure auch bei der Tumeszens-
Lokalanästhesie im Rahmen von Liposuktionen. „Dieser Eingriff wird heute auch in der Leitlinie zur Behandlung des Lipödems empfohlen“, so Fratila. Durch die Ko-Applikation dieser Substanz werde das Gesamtvolumen und der schmerzunempfindliche Bereich vergrößert und zwar ohne dass dadurch die Wirkdauer der Anästhesie oder die Wundabheilung beeinträchtigt wird.

Ein weiteres Indikationsgebiet für die Hyaluronidase ist die Korrektur von hypertrophen Narben im Gesicht. „Vorteilhaft dabei ist, dass das Enzym die Bildung von Hyaluronsäure mit geringem Molekulargewicht katalysiert und diese kleinen Moleküle stimulieren die Proliferation von mesenchymalen Stammzellen und die Angiogenese“, so Prof. Uwe Wollina, Dresden.

Dr. Peter Stiefelhagen

Pressekonferenz "Update Hyaluronidase: Anwendungsspektrum in der ästhetischen Dermatologie" im Rahmen der 25. Fortbildungswoche für praktische Dermatologie und Venerologie, München, 27.7.2016; Veranstalter: Riemser Pharma

\section{Wundbehandlung}

\section{Antimikrobielle Peptide für die Wundbehandlung}

Antimikrobielle Peptide unterscheiden sich von herkömmlichen Antibiotika in vielerlei Hinsicht - allen voran durch eine geringe Tendenz zur Ausbildung von Resistenzen. In der Therapie von Wunden findet das antimikrobielle Peptid (AMP) Tyrothricin (Tyrosur ${ }^{\circledR}$ ) Anwendung. Der Wirkstoff umfasst mit seinem Wirkspektrum die wichtigsten Erreger von Wundinfektionen, zeigt dabei eine gute antimikrobielle Effektivität sowie Verträglichkeit und fördert zugleich die Wundheilung. Er ist zugelassen zur Behandlung von kleinflächigen, det werden sie von Keratinozyten, Epithelzellen der ekkrinen Schweißdrüsen, Sebozyten sowie Mastzellen und Leukozyten.

Der Wirkstoff Tyrothricin zeigt eine effektive wie rasche bakterizide Wirksamkeit mit einem breiten Wirkspektrum, das grampositive Bakterien inklusive MRSA, einige gramnegative Bakterien und verschiedene Pilzarten umfasst. Dabei ist er gut verträglich und zeigt -

Mit der richtigen Wundversorgung schnell vergessen.

$+$
oberflächlichen, wenig nässenden Wunden mit bakterieller Superinfektion mit Tyrothricin-empfindlichen Erregern, wie z. B. Riss-, Kratz- und Schürfwunden.

AMP werden von allen Organismen gebildet, bei Menschen stellen sie u.a. einen wichtigen Schutzmechanismus der Haut und Schleimhäute gegen Mikroorganismen dar. Gebil-

ge-

$$
\text { (chnell ve }
$$

verglichen mit antiseptisch wirkenden Mitteln - keine negativen Eigenschaften auf körpereigenes Gewebe. Vielmehr ist das Gegenteil der Fall: Denn für Tyrothricin konnten in klinischen Untersuchungen wundheilungsfördernde Effekte - gemessen an einer beschleunigten Heilung sowie einer deutlichen Besserung der Symptomatik - nachgewiesen werden.

Die Anwendung eines Hydro-Gels bringt weitere Vorteile für die Wundheilung mit sich. Das Gel sorgt für eine intensive Befeuchtung des verletzten Hautareals und fördert mit ihrer luftdurchlässigen Konsistenz den Gasaustausch zwischen Wunde und Umgebung. „Es ist im Speziellen ihr breites Einsatzspektrum, das Hydro-Gele zu einer Grundlage der ersten Wahl macht", erklärt Prof. Joachim Dissemond, Essen, und ergänzt: „Bei akuten Wunden empfehle ich die Anwendung von der Wundreinigung bis zur Epithelisation, bei chronischen Wunden vor allem in den beiden ersten Wundheilungsphasen." In Tyrosur ${ }^{\circledast} \mathrm{Gel}$ sind die Vorteile einer Hydro-Gel-Grundlage und des antimikrobiellen Wirkstoffes Tyrothricin vereint.

red 